\title{
Farmland WSN Incremental Deployment Strategy Based on Signal Attenuation Model
}

\author{
Dayong Liu ${ }^{1,2, \text { a }}$,Chunjiang Zhao ${ }^{2,3, b^{*}}$, Huarui $\mathrm{Wu}^{2,3, \mathrm{c}}$ and Feifei $\mathrm{Li}^{2,3, \mathrm{~d}}$ \\ ${ }^{1}$ College of Computer Science and Technology, Beijing University of Technology, Beijing, China \\ ${ }^{2}$ Beijing Research Center for Information Technology in \\ Agriculture, Beijing Academy of Agriculture and Forestry Sciences, Beijing, China \\ ${ }^{3}$ National Engineering Research Center for Information Technology in Agriculture, Beijing, China

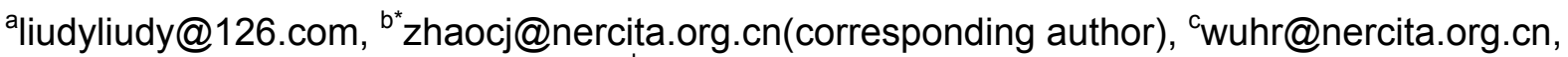 \\ ${ }^{d}$ liff@nercita.org.cn
}

Keywords: wireless sensor network, signal attenuation model, incremental deployment, farmland

\begin{abstract}
This article discusses the partition node incremental deployment method in the wireless sensor network, which is divided into partition structure deployment, incremental deployment nodes and node position adjustment. It suggests how the partition node incremental deployment method might influence the running of the farmland wireless sensor networks, and gives illustration of how the connective and stable communication were provided against the instability of the farmland wireless sensor networks.
\end{abstract}

\section{Introduction}

Node deployment is the basis of topology construction, route selection and data transmission for the wireless sensor networks (WSN). It is mainly focused on improving the coverage rate of monitoring area, network connectivity, reducing the cost of node configuration, extending the network lifetime, etc. It is very important that we consider the node deployment, for the indicators restrain each other.

Most of the research on nodes deployment is based on a supposed open and stable wireless signal transmission environment ${ }^{[1]}$. The height of crops have great influence on the radio signal propagation. The traditional method can't meet the need of non-uniform space transmission ${ }^{[2]}$.

Based on the signal attenuation model, the article suggests a partition node incremental deployment method (PNIDM) for WSN, which is applied to the farmland environment monitoring. PNIDM can provide the connective and stable communication by relatively small number of nodes.

\section{Related Works}

The method is divided into static and dynamic deployment. The static method is not suitable for large scale application. The efficiency of random method is high, but the distribution of nodes is uncertain. The method can't provide the performance of network monitoring and communication stability ${ }^{[3]}$.

Node deployment is the process of solving a large-scale combinational optimization. In recent years, the efficient approximation algorithm for solving large combinatorial optimization problems has been paid to great attention, such as the glowworm swarm optimization algorithm, particle swarm optimization, simulation annealing genetic algorithm etc. ${ }^{[4]}$. In the literature ${ }^{[5]}$, the method of $\mathrm{K}$-Coverage is used to ensure the connectivity by increasing the number of nodes.

The key problem of is how to deploy the nodes in the sensing area. A distributed scalable WSN is proposed in the literature ${ }^{[6,7]}$. PFA algorithm assumes that each node receives a close gravitational repulsion and other remote nodes to other nodes through the balance between the forces that stabilize each node in the right position. When a new node is added, the nodes need re-position to balance by using PFA algorithm ${ }^{[8]}$. Virtual Force Algorithm (VFA) is the relationship between the distance and the position of the node and the node, the node and the obstacle, and the virtual force algorithm is transformed into the virtual attraction and repulsion force. Each node is subjected to a number of 
attractive and repulsive forces, they form a joint force to promote the node to move and keep their balance.

Above- mentioned studies improve the network coverage of wireless sensor networks, and provide the connectivity and long time working of the network. However, these algorithms ignore the wireless transmission environment in the monitoring scene. As a result, these nodes deployment algorithms are lack of adaptability in the practical application of farmland wireless sensor networks.

\section{Zone Partition and Incremental Deployment}

The method proposed in this paper is a combination of static and dynamic deployment. The static deployment can avoid the uneven distribution of random deployment. The dynamic deployment, avoiding the deployment of large-scale farmland environment in the operational environment, reduces the difficulties in the deployment.

Node deployment method is shown in Fig. 1. First of all, partition structure deployment method is used in the entire monitoring area. Secondly, the number of nodes is increased. Finally, the location of the node is removed mildly according to the different regions of the wireless signal transmission environment.

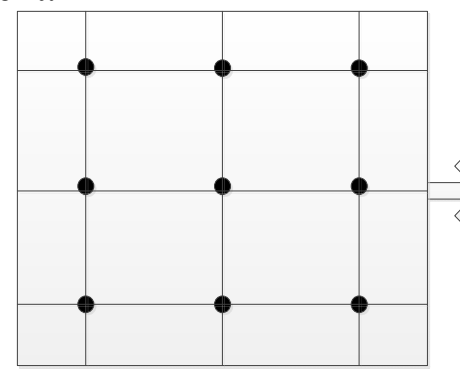

(a) Partition Structured Deployment

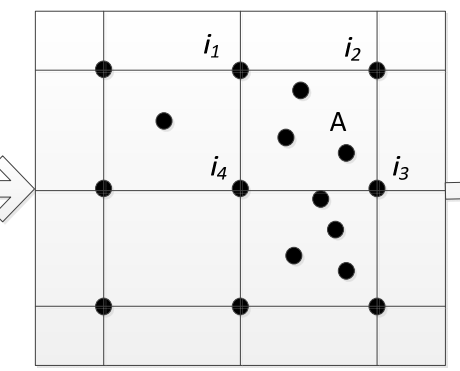

(b) Node Supplement

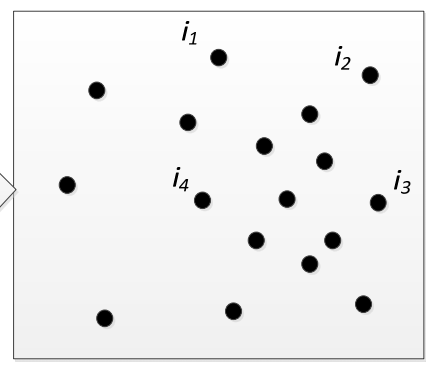

(c) Node Position Adjustment

Fig.1 Node Deployment Diagram

Partition Structured Deployment. The entire monitoring area is divided into a number of small structural areas. According to the communication data of the structured deployment nodes, the environment of the wireless signal transmission in a certain area is predicted based on the environment channel model.

A structured deployment is used in a wide range. The layout number of the positive quadrilateral is $n$, the marked area of the single sub region is $r_{c}^{2}$. Each node sends message, including message type, node ID, battery energy, time, etc. to all neighbors in the $r_{c}$ range with maximum power. When the neighbor node receives the broadcast message, the RSSI and other information are stored.

After broadcasting $m_{0}$ receiving a M2 type message, the completed signal should be sent by the node. The node calculates and obtains power message on the basis of the temporary storage of information receiving between adjacent nodes on the rate and the average received power calculation.

According to the characteristics of signal transmission between nodes, sink nodes predict the transmission environment in each cell. Parameter $p_{r d c}(i, j)$ which indicates the time from node $i$ to node $\mathrm{j}$ radio signal communication situation is defined as follows:

$$
p_{r d c}(i, j)=\frac{m}{m_{0}} \times \frac{R S S I_{\text {average }}-R S S I_{v}-10 \lg P_{t}}{-R S S I_{v}-10 \lg P_{t}}
$$

where $R S S I_{\text {average }}$ represents the average received power, $m$ represents the total number of successful received packets, $m_{0}$ represents the total number of packets sent by the node $\mathrm{i}, R S S I_{v}$ represents node $\mathrm{j}$ receiver sensitivity, $P_{t}$ represents node i transmission power.

Node Supplement. The number of nodes is related to the communication supplement in all regions in every sub-region. The number of nodes is increased in order to maintain network connectivity. If the 
communication is better, the less supplied nodes are need. According to the average value $\left(\bar{p}_{\text {rdc }}\right)$ of every partition $\left(p_{r d c}(i, j)\right)$ and every partition area, the number of complement nodes is calculated.

$$
\bar{p}_{r d c}(A)=\frac{\sum_{i=1}^{4} \sum_{j=1, i \neq j}^{4} P_{r d c}(i, j)}{4 \times 3}
$$

Node Position Adjustment. First, nodes are added by random method in the sub-region. Secondly, as definition 4 , the signal strength from neighbor nodes is calculated.

$$
d_{R S S I}(i, j)=\frac{d(i, j)}{\bar{p}_{r d c}(A)}
$$

By the signal propagation model, we can estimate the desired signal strength distance $\left(d_{\theta}\right)$. When the distance of actual signal strength between nodes is greater than $d_{\theta}$, there suppose to be a gravitational correlation between the nodes. On the contrary, when the distance of actual signal strength between nodes is lower than $d_{\theta}$, we think there is repulsive force within the nodes. According interactive force within the neighboring nodes, the node changes its position, as shown Fig. 2 .

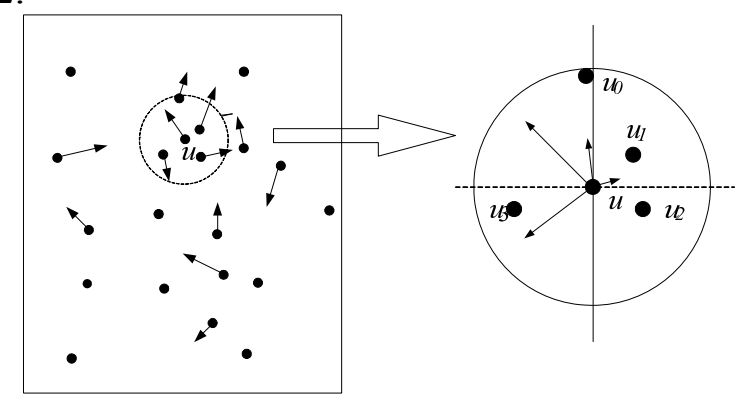

Fig.2 Node Virtual Force Analysis

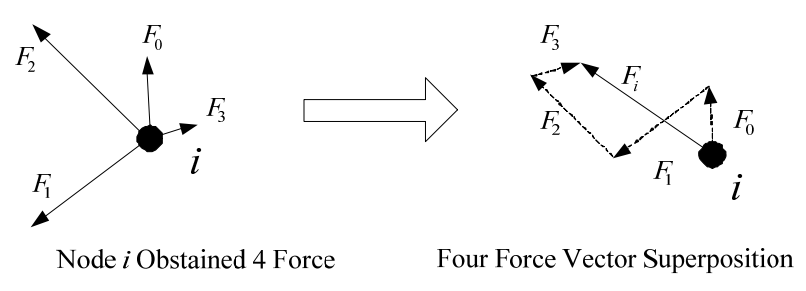

Fig.3 Node Force Analysis Diagram

If $d_{R S S I}(i, j)-d_{\theta}(i) \geq 0$, node i obtains the interactive force of node $\mathrm{j}$. If $d_{R S S I}(i, j)-d_{\theta}(i) \leq 0$, node $i$ obtains the reversed force of node $j$. The node $u$ receives the force of a number of neighbor nodes, which is the vector sum of the other neighbor nodes forces.

$$
\vec{F}=\vec{F}_{0}+\vec{F}_{1}+\ldots+\vec{F}_{s}
$$

As shown Fig. 3, each node is based on the signal strength of its neighbor nodes and the distance of the signal strength of the node in the region, and the size and direction of the resultant force is calculated by the formula 3 and 4 . Each node adjusts its position under the action of the resultant force. In the process of adjustment, the force of the node is changed. When the nodes in the network are adjusted to achieve the state of the force balance through the position, the layout of the nodes is completed.

\section{Analysis}

Experimental Scheme. In this experiment, the the CC1101EMK433 module produced by TI company is used. In order to verify the effectiveness of the proposed algorithm, a $100 \mathrm{~m} \times 100 \mathrm{~m}$ corn field is set as the experimental area. The first through the corn field area zoning rules for deployment, the deployment of the quadrangle side is $15 \mathrm{~m}$. Secondly, according to the situation of farmland signal attenuation, the incremental deployment and node position adjustment are carried out. We obtain the packet loss probability(PLP) is $10 \%$, and $R S S I_{\text {average }}$ is $-45 \mathrm{dbm}$. 
Comparative Analysis. According to the formula 2, the $\bar{p}_{r d c}(A)$ is calculated as 0.08 . When the coefficient $a$ is 0.1 , the square rule partition needs to be supplemented with 44 nodes, and 80 nodes are deployed.

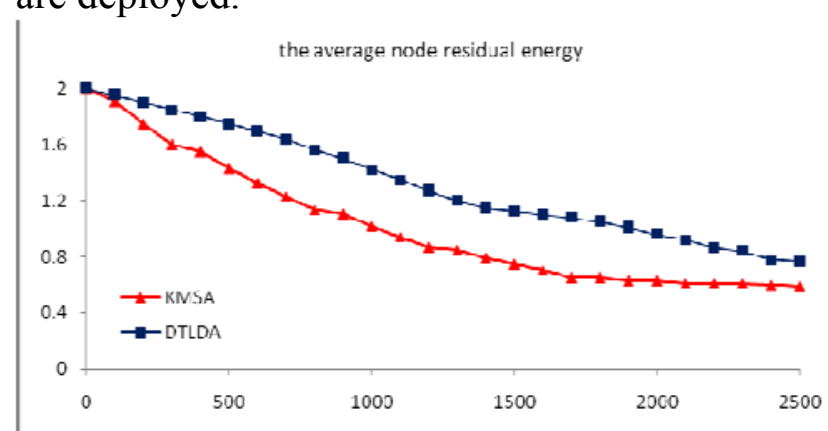

Fig.4 Average node residual energy

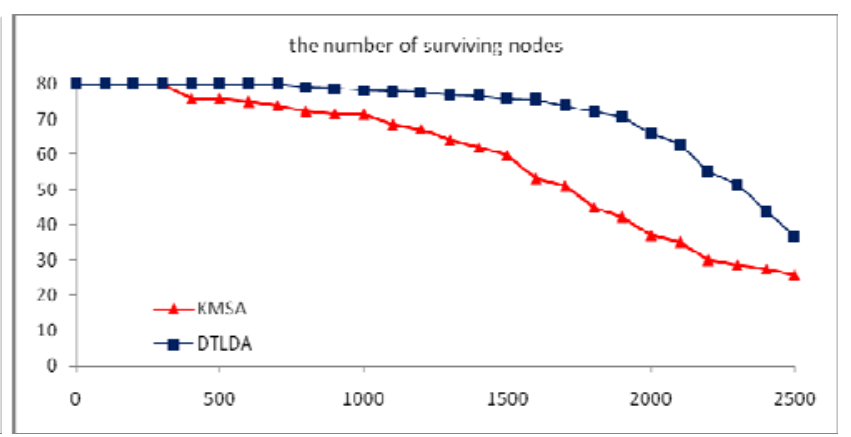

Fig5. Number of alive nodes

Fig. 4 is the comparison of average node residual energy between two algorithms, and Fig. 5 is the comparison of number of surviving nodes. As it is shown in Fig. 4 the average power consumption of DTLDA is lower than KMSA. In Fig. 5 the number of dead nodes of DTLDA is smaller than KMSA in the first 2000 rounds, but quickly increases to about 500 in 500 rounds. This means DTLDA has a good performance in the power balance between nodes. According to the results, DTLDA has a better performance than KMSA.

\section{Conclusion}

Based on the wide range of agricultural environment and uneven coverage of the network communications, a partition node incremental deployment strategy is proposed. Experimental results show that this method can be greatly maintained the connection within the nodes. In the future, the hybrid deployment method of solar and battery-powered nodes should be studied due to the farmland environmental monitoring requirements.

\section{Acknowledgements}

This work was financially supported by Natural Science Foundation of China $(61271257,61471067$, 61571051).

\section{References}

[1] Toumpis S, Tassiulas L. IEEE Transactions on Information Theory Vol. 52(2006), p. 2935-2953.

[2] An W, Song L. Wireless Communications \& Mobile Computing Vol.15(2015), p. 1606-1620

[3] Lu Z K, Huang L S , Wan Y Y, et al. Mini-Micro Systems, Vol.27(2016), p. 2003-2006.

[4] Srivastava J R, Sudarshan T S B. Soft Computing Vol.19(2015), p. 1-14.

[5] Gupta H P, Rao S V. Intelligent Transportation Systems IEEE Transactions Vol.16(2015), p. 1861-1871.

[6] Heo N, Varshney P K. A Distributed Self-Spreading Algorithm for Mobile Wireless Sensor Networks [C]// Proc. IEEE Wireless Communications and Networking Conf. New Orleans: LA, 2003:1597-1602.

[7] Truong T T, Brown K N and Sreenan C J. Ad Hoc Network Vol.33(2015), p. 190-208

[8] Le Berre M, Rebai M. Wireless Personal Communications Vol.84(2015), p. 2187-2213 